\title{
Muscle function impairment in cancer patients in pre-cachexia stage
}

\author{
Stefania Dalise (1), Peppino Tropea (2), Luca Galli (3), Andrea Sbrana (3), Carmelo \\ Chisari (1) \\ (1) Unit of Neurorehabilitation, University Hospital of Pisa, Pisa, Italy; (2) Department of \\ Neurorehabilitation Sciences Casa Cura Policlinico, Milan, Italy; (3) Unit of Oncology 2, \\ University Hospital of Pisa, Pisa, Italy.
}

This article is distributed under the terms of the Creative Commons Attribution Noncommercial License (CC BY-NC 4.0) which permits any noncommercial use, distribution, and reproduction in any medium, provided the original author(s) and source are credited.

\begin{abstract}
Cancer cachexia has been reported to be directly responsible for at least $20 \%$ of cancer deaths. Management of muscle wasting in cancer-associated cachexia appears to be of pivotal importance for survival of patients. In this regard, it would be interesting to identify before its patent appearance eventual functional markers of muscle damage, to plan specific exercise protocols to counteract cachexia. The muscle function of 13 oncologic patients and 15 controls was analyzed through: i) analysis of the oxidative metabolism, indirectly evaluated trough dosage of blood lactate levels before and after a submaximal incremental exercise on a treadmill; ii) analysis of strength and, iii) endurance, in both lower and upper limbs muscles, employing an isokinetic dynamometer. Statistical analyses were carried out to compare the muscle activities between groups. Analysis of oxidative metabolism during the incremental exercise on a treadmill showed that patients performed a shorter exercise than controls. Lactate levels were significantly higher in patients both at baseline and after the task. Muscle strength analysis in patients group showed a reduction of Maximum Voluntary Contraction during the isometric contraction and, a tendency to fatigue during endurance task. Data emerging from this study highlight an impairment of muscle oxidative metabolism in subjects affected by a pre-cachexia stage of cancer. A trend of precocious fatigability and an impairment of muscle strength production were also observed. This evidence underlines the relevance of assessing muscle function in order to develop novel rehabilitative approaches able to counteract motor impairment and eventually to prevent cachexia in these patients.
\end{abstract}

Key Words: Cachexia, Oxidative Metabolism, Cancer, Muscle Modifications, Physical Exercise

Eur J Transl Myol 30 (2): 258-267, 2020

Cachexia is one of the most frequent effects of malignancy, affecting $50 \%$ of untreated cancer patients. $^{1,2}$ Cancer-associated cachexia (CC) is a metabolic syndrome characterized by loss of skeletal muscle with or without loss of fat mass. ${ }^{3}$ This syndrome has been reported to be directly responsible for at least $20 \%$ of cancer deaths. ${ }^{4}$ Decrease in muscle mass leads to generalized weakness, decreased mobility, and compromised quality of life (QoL). Despite its association with devastating chronic diseases, such as cancer, the clinical importance of cachexia has been neglected for decades. It is now established that cachexia affects prognosis, and reduces the response to therapy. ${ }^{1}$ Recently, it has emerged that skeletal muscle mass is affected not only by disease progression but also by both therapeutic and palliative treatments. It has been suggested that chemotherapy may be a major contributor to the development and sustainment of CC. ${ }^{5}$ The newly defined diagnostic criteria of $\mathrm{CC}$ will help diagnosis and clinical management, as well as basic research. ${ }^{3}$ To date, effective treatments for cachexia are limited. Ghrelin agonists, such as anamorelin, demonstrated efficacy in patients with metastatic non-small cell lung cancer and cachexia, ${ }^{6-7}$ with limited and short-lasting efficacy. Although molecular mechanisms of $\mathrm{CC}$ remain yet to be completely defined, research in the last two decades has made significant progress. Firstly, several mediators have been identified, including proinflammatory cytokines, such as tumor-necrosis factor alpha (TNF $\alpha$ ), interferon gamma (IFN $\gamma$ ) and interleukin-6 (IL-6), as well as tumorsecreted Proteolysis-Inducing Factor (PIF). ${ }^{1-8-9}$ Secondly, cachexia mediators reduce lean muscle mass by altering homeostasis between synthesis and degradation of muscle proteins. ${ }^{10-12}$ Protein degradation is largely regulated by the lysosomal and ubiquitin-proteasome 


\section{Muscle impairment in pre-cachexia cancer patients}

Eur J Transl Myol 30 (2): 258-267, 2020

pathways. ${ }^{13-16}$ Genes regulating proteasome-mediated proteolysis are elevated in CC animal models as well as in patients. ${ }^{17-18}$ Identification of two muscle-specific E3 ubiquitin ligases, Muscle RING-finger protein (MuRF1) and atrogin-1/muscle atrophy F-box (MAFbx), have further demonstrated the predominant contribution of the proteasome pathway in muscle wasting. ${ }^{19-20}$ Despite these insights into the molecular aetiology of CC, little is known about muscle wasting impact on muscle function. Significant loss of muscle mass leads to generalized muscle weakness, however, little is known about other aspects of muscle function, such as force-velocity characteristics, power output, metabolic properties, and fatigability. Various interventions have been used to counteract body weight loss in cachexia, including aggressive nutritional support and administration of appetite stimulants. Often, these approaches, resulted in weight gain due to water retention or fat gain, uncoupled by functional body lean mass gain. ${ }^{1}$ Contractile activity is necessary for postnatal muscle growth and the maintenance of muscle mass in adults, and increased work can cause fiber hypertrophy. ${ }^{21}$ Conversely, disuse or denervation causes rapid atrophy. ${ }^{22}$ It has been shown that muscle contraction performed immediately after immobilization rapidly instigates profound changes in gene expression collectively linked to the suppression of muscle catabolism and the promotion of muscle hypertrophy and remodelling, with a decline in MAFbx and MuRF1. In literature, several studies have investigated the beneficial effects of physical exercise in animal models of cancer. In a recent study, Aulino and colleagues, ${ }^{23}$ observed that physical activity diminishes total body weight loss of tumor-bearing mice and increases survival. Authors related these effects to significant changes in levels of hormones namely arginine vasopressin (AVP) and ghrelins (i.e., acyl ghrelin (AGHR) and des-acyl ghrelin (DGHR) that are known to be potent pro-myogenic and anti-cachexia factors. Recently, two epidemiological studies and one clinical trial showed that physical activity performed post-diagnosis, rather than their exercise habits before disease onset, improves prognosis in cancer patients. ${ }^{24-26}$ These studies analyzed cohorts of cancer patients and concluded that physical activity after diagnosis decreases the risk of both cancer-specific and overall mortality. ${ }^{24-26}$ The limits of these observations are that the studies are observational in nature and cause and effect cannot be assumed. In this regard, it would be interesting to identify early functional markers of muscle damage in order to plan specific exercise protocols to counteract CC. For this purpose in our study, we have extensively analyzed muscle function in a heterogeneous group of cancer patients at a different stage of the disease.

\section{Materials and Methods}

The patients involved in the study were enrolled at the Department of Clinical Oncology, University of Pisa. All patients with a cancer diagnosis (of any type and any stage) who were about to start chemotherapy for their disease were eligible for the inclusion in the study. All participants to the study were evaluated for the presence of signs of symptoms of pre-cachexia or cachexia, according to the criteria emerged from the international consensus conference published in $2011,{ }^{27}$ as the major exclusion criterion. Other exclusion criteria for both groups were: a severe pulmonary or cardiac failure, fever, neurologic/muscular illnesses or a physical inability to exercise. No limits on eligibility were based on the sex of participants neither on their age. Thirteen patients affected by solid tumors were included in the study. The patient group was heterogeneous in terms of demographic and anthropometric characteristics (e.g., age, sex, weight, height), stage of the disease and received treatments (e.g., surgery, chemotherapy, or radiotherapy). Fifteen healthy subjects were recruited as a control group, matched for demographic and anthropometric characteristics (i.e., age, sex, weight, height) to patients. After a careful explanation of procedures, informed consent was obtained from all the subjects. All research procedures were in accordance with the Declaration of Helsinki. The study was approved by the local Ethical Committee of University Hospital of Pisa. All the evaluations described below were performed, for the patient group, the very same day or, at most, the day before, they started their chemotherapy. The muscle oxidative efficiency, the maximum voluntary strength of a distal and a proximal district and the resistance to exercise were assessed in both groups (i.e., patients and controls), and described extensively in next paragraphs. In addition, the patients' Body Mass Index (BMI) was evaluated, and calculated according to the following formula:

$$
B M I=\frac{W}{H^{2}}
$$

where $\mathrm{W}$ is the weight of subjects express in kilograms (kg) and $\mathrm{H}$ the height of subjects express in meters (m).

\section{Muscle oxidative efficiency evaluation}

Muscle oxidative efficiency was indirectly evaluated through the determination of blood lactate levels before and after an aerobic exercise on a treadmill: this test provides an estimation of energetic consumption during the motor task. ${ }^{28-29}$ The exercising test consisted of an incremental submaximal exercise on a calibrated electronically braked treadmill. Exercise protocol consists of 24 minutes of walking at a constant speed of $3 \mathrm{~km} / \mathrm{h}$; the first and last minutes were, respectively, warm-up and cool-down periods. During the first three minutes, the inclination of the treadmill was $0^{\circ}$ then it increased gradually of $2.5 \%$ slope every two minutes. The exercise was early stopped if the patient reached $75 \%$ of maximal heart rate so keeping the exercise predominantly aerobic. The maximal heart rate ( $\left.\mathrm{HR}_{\mathrm{MAX}}\right)$ was theoretically calculated according to the formula: $\mathrm{HR}_{\mathrm{MAX}}=208-0.7 \mathrm{x}$ age. ${ }^{30}$ All subjects were informed that they could stop exercise at any time. Lactate was 
assessed through venous blood samples, collected before $\left(\mathrm{t}_{0}\right)$, immediately after $\left(\mathrm{t}_{1}\right)$ and 5,10 and 30 minutes after the end of the exercise (respectively: $t_{2}, t_{3}, t_{4}$ ). Blood sample was collected in EthyleneDiamineTetraAcetic Acid (EDTA) tubes. Samples were withdrawn by venipuncture. Lactate was rapidly analyzed through an enzymatic assay.

\section{Muscle strength evaluation}

Muscle strength was evaluated measuring maximum voluntary contraction (MVC) in an isometric mode in both lower (knee extension) and upper (handgrip) limbs. Tests were performed on an isokinetic dynamometer (PrimusRS Multi-Joint System dynamometer, BTE Technologies). For evaluation of knee extension, patients sat on a chair with their upper body strapped firmly against the backrest of the chair, straps tightened across their thighs, arms were folded. Hip and knee angles were set respectively at $110^{\circ}$ and at $120^{\circ}\left(180^{\circ}\right.$ refers to full extension). For evaluation of handgrip, the subject sat on the chair with their shoulders at $0^{\circ}$ of abduction and neutral rotation, elbows unsupported at $90^{\circ}$ flexion and forearms in the neutral position. Before starting test sections, subjects were instructed about the correct procedure to exert maximum voluntary contraction, as hard as possible without moving the rest of the body, and to maintain it for $3 \mathrm{~s}$. Each subject performed three consecutive repetitions for each limb and each side. A rest time (10 s) was observed between consecutive trials. The three tests were accepted when the coefficient of variation $(\mathrm{CV})$ was less than $10.0 \% .^{31}$

\section{Muscle fatigue and muscle recovery evaluations}

Muscle fatigue was evaluated measuring sustained contractions in isometric tasks, in both lower (knee extension) and upper (handgrip) limbs. Endurance tests were performed on an isokinetic dynamometer (see above for further details). Subject, seated as described above, was instructed to exert MVC for knee extension and for handgrip and to maintain it for $60 \mathrm{~s}$. Both sides were evaluated. Each subject was instructed about the test before starting and was not further encouraged during the performance. An index of muscle fatigue was extracted as the slope of the linear regression of contraction trend. To compare them, the endurance index was normalized for the MVC. To evaluate an additional parameter, the muscle recovery, the MVC in isometric mode (with $3 \mathrm{~s}$ hold time) was performed after $20 \mathrm{~s}$ of rest at the end of muscle fatigue trial. To obtain a single explicative parameter the difference $(\Delta)$ between MVC in muscle recovery time and the strength exerted in the last $3 \mathrm{~s}$ of muscle fatigue task was calculated.

\section{Statistical analysis}

Data are presented as mean with standard deviation. The significance of statistical tests was set at $\alpha=0.05$. Distributions were checked for normality with a onesample Kolmogorov-Smirnov test. The homoscedasticity of residuals was tested with Levene's test. The normality test showed that data did not fit the normality distribution $(p<0.05)$ and Levene's test showed that all homologous datasets were characterized by equal variance. Since the ANalysis Of VAriance (ANOVA) is relatively robust for violations of the normality assumption and more sensitive to heterogeneous variances, ${ }^{32}$ the preliminary analysis of the data samples allowed the authors to confidently use the ANOVA as the main statistical test in this study. Due to non-normality of the dataset, Wilcoxon test was used to compare walking time on the treadmill (i.e., duration), and both muscle strength (i.e., MVC in isometric mode), fatigue parameters (i.e., slope and muscle recovery after $20 \mathrm{~s}$ of resting time (i.e., $\Delta$ )) in patients and control group, for knee extension value and handgrip measurements. In the analysis of muscle

Table 1 Demographic and anthropometrics of cancer patients participants.

\begin{tabular}{ccccccc}
\hline ID & $\begin{array}{c}\text { Age } \\
(\mathbf{y r})\end{array}$ & Sex & $\begin{array}{c}\text { Weight } \\
(\mathbf{k g})\end{array}$ & $\begin{array}{c}\text { Height } \\
(\mathbf{c m})\end{array}$ & $\begin{array}{c}\text { BMI } \\
\left(\mathbf{k g} / \mathbf{m}^{\mathbf{2}}\right)\end{array}$ & Level of physical exercises \\
\hline P01 & 35 & M & 107 & 190 & 29.6 & Professional basket player \\
P02 & 54 & M & 66 & 175 & 21.6 & Mildly active \\
P03 & 69 & M & 83 & 167 & 29.8 & Sedentary \\
P04 & 31 & M & 82 & 183 & 24.5 & Active \\
P05 & 33 & M & 74 & 174 & 24.4 & Active \\
P06 & 43 & M & 97 & 180 & 29.9 & Active \\
P07 & 23 & M & 70 & 180 & 21.6 & Professional football player \\
P08 & 69 & F & 69 & 168 & 24.4 & Sedentary \\
P09 & 58 & M & 72 & 180 & 22.2 & Moderately active \\
P10 & 48 & M & 69 & 172 & 23.3 & Mildly active \\
P11 & 30 & M & 96 & 196 & 25.0 & Active \\
P12 & 28 & M & 70 & 178 & 22.1 & Active \\
P13 & 32 & M & 70 & 180 & 21.6 & Active \\
\hline
\end{tabular}

Labels in $3^{\text {rd }}$ column refer to: F: Female; M: Male. 
Table 2. Summary of main clinical features of cancer patients recruited in this study.

\begin{tabular}{|c|c|c|c|c|c|}
\hline ID & Cancer Location & Comorbidities & Medications & $\begin{array}{l}\text { Time elapsed } \\
\text { from diagnosis } \\
\text { (months) }\end{array}$ & Treatment \\
\hline P01 & Mediastinal + & - & - & 3 & $\mathrm{CT}$ \\
\hline P02 & Lung + & COPD & BAA & 24 & $\mathrm{CT}+\mathrm{RT}$ \\
\hline P03 & Pancreatic + & AHT & $\mathrm{ACEi}$ & 2 & $\mathrm{CT}$ \\
\hline P04 & Testicular + & - & - & 22 & $\mathrm{ST}+\mathrm{CT}$ \\
\hline P05 & Testicular & - & - & 4 & $\mathrm{ST}+\mathrm{CT}$ \\
\hline P06 & Testicular + & - & - & 24 & $\mathrm{ST}+\mathrm{CT}$ \\
\hline P07 & Testicular & - & - & 1 & $\mathrm{ST}+\mathrm{CT}$ \\
\hline P08 & Colon + & $\mathrm{H}$ & Statins & 3 & CT \\
\hline P09 & Testicular + & - & - & 61 & $\mathrm{ST}+\mathrm{CT}$ \\
\hline P10 & Retromandibular + & - & - & 12 & $\mathrm{ST}+\mathrm{CT}+\mathrm{RT}$ \\
\hline P11 & Testicular & - & - & 3 & ST \\
\hline P12 & Testicular & - & - & 2 & $\mathrm{ST}+\mathrm{CT}$ \\
\hline P13 & Testicular & - & - & 3 & $\mathrm{ST}+\mathrm{CT}$ \\
\hline
\end{tabular}

Labels in $2^{\text {nd }}, 3^{\text {rd }}, 4^{\text {th }}$, and $6^{\text {th }}$ columns refer to: + : presence of metastasis; COPD: Chronic obstructive pulmonary disease; AHT: Arterial Hypertension; H: Hypercholesterolemia; BAA: Beta-adrenergic agonist; ACEi: Angiotensinconverting enzyme inhibitors; CT: Chemotherapy; RT: Radiotherapy; ST: Surgical Therapy.

oxidative efficiency, a two-way ANOVA of "group" (i.e., patients vs. control group) and "time" (i.e., $\mathrm{t}_{0}, \mathrm{t}_{1}, \mathrm{t}_{2}$, $t_{3}, t_{4}$ ) with repeated measures on "time" was used to find significant differences in the lactate blood concentrations. Post-hoc an unpaired t-test with Bonferroni correction $(\alpha=0.05 / 5)$ was applied to identify pairwise differences. Data processing and statistical analysis were performed using custom-written MATLAB (The MathWorks, Inc., Natick, MA, USA) scripts.

\section{Results}

Twelve males and one female were enrolled (age $42.5 \pm$ 15.7 years, range 23-69 years). Clinical characteristics of the included patients are summarised in Table 1 and Table 2. No relevant comorbidities were present and enrolled patients have active to moderately-active lifestyle. Patients' anamnestic data did not show any weight loss (larger than 5\%) in the 12 months before the enrolment for this study. All patients had no signs of

Table 3. Demographic and anthropometrics of controls.

\begin{tabular}{lcccccc}
\hline ID & $\begin{array}{c}\text { Age } \\
(\mathbf{y r})\end{array}$ & Sex & $\begin{array}{c}\text { Weight } \\
(\mathbf{k g})\end{array}$ & $\begin{array}{c}\text { Height } \\
(\mathbf{c m})\end{array}$ & $\begin{array}{c}\text { BMI } \\
\left(\mathbf{k g} / \mathbf{m}^{2}\right)\end{array}$ & Level of physical exercises \\
\hline C01 & 22 & M & 70 & 185 & 20,5 & Active \\
C02 & 30 & M & 75 & 175 & 24,5 & Professional football player \\
C03 & 40 & M & 78 & 177 & 24,9 & Active \\
C04 & 73 & F & 60 & 160 & 23,4 & Mildly active \\
C05 & 73 & M & 59 & 168 & 20,9 & Moderately active \\
C06 & 33 & M & 95 & 185 & 27,8 & Weightlifters \\
C07 & 43 & M & 86 & 170 & 29,8 & Active \\
C08 & 52 & M & 92 & 180 & 28,4 & Sedentary \\
C09 & 52 & F & 79 & 165 & 29,0 & Active \\
C10 & 77 & M & 70 & 178 & 22,1 & Mildly Active \\
C11 & 37 & M & 76 & 178 & 24,0 & Active \\
C12 & 59 & M & 75 & 173 & 25,1 & Moderately active \\
C13 & 57 & F & 70 & 168 & 24,8 & Active \\
C14 & 59 & M & 88 & 175 & 28,7 & Moderately active \\
C15 & 57 & M & 105 & 182 & 31,7 & Sedentary \\
\hline
\end{tabular}

Labels in $3^{\text {rd }}$ column refer to: F: Female; M: Male. 


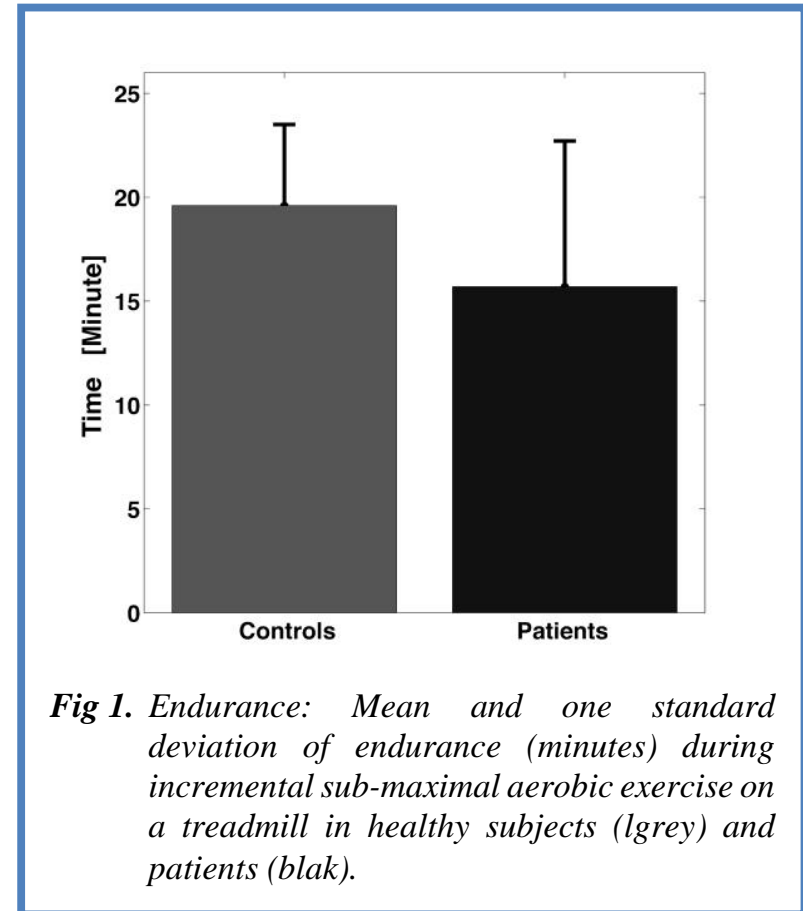

cachexia nor pre-cachexia. For each patient, the BMI was greater than $20 \mathrm{~kg} / \mathrm{m}^{2}(24.6 \pm 3.2)$ (see Table 1). Twelve males and three females were enrolled as a control group (Table 3). There were no significant differences between patients and controls both for age and BMI (age: $49.1 \pm$ 17.5 years, range $22-77$ years; BMI: $25.7 \pm 2.8$ ). All subjects included in the study performed the required tasks without reporting any side effects. Regarding the lactate test, generally healthy subjects walked on treadmill longer than patients (healthy subject: $19.6 \pm 3.9$ minutes vs. patients: $15.7 \pm 7.7$ minutes) (Figure 1 ). The

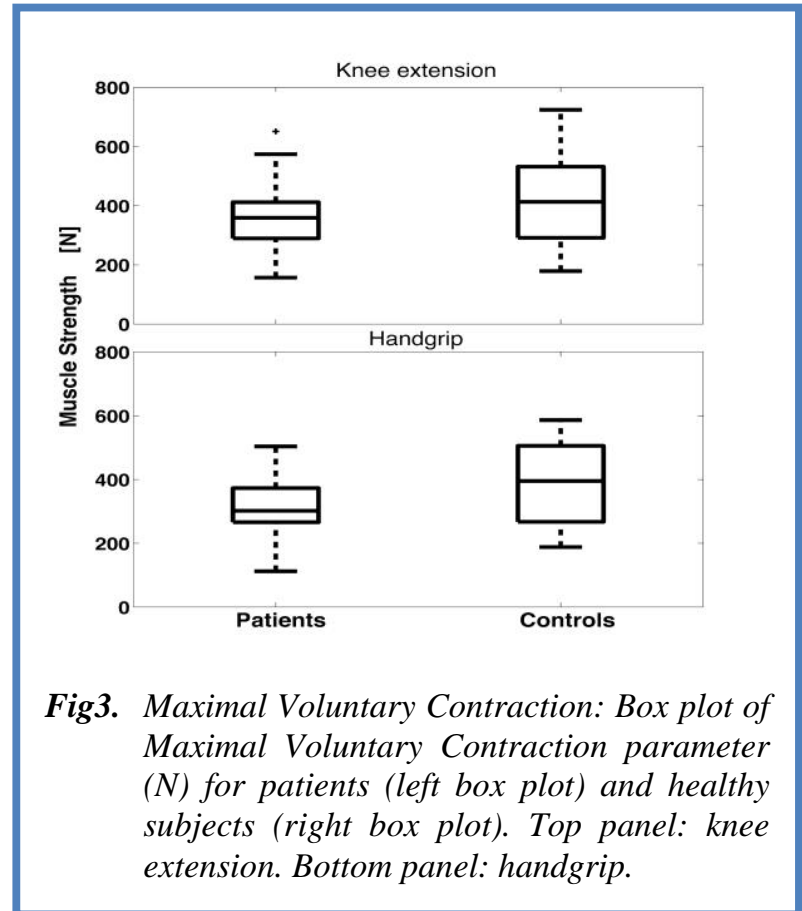

blood concentration of lactate increased during the exercise and decreased during the recovery phase in both groups (i.e., patients and controls). Two-way ANOVA revealed a highly significant difference in both factors ("group" and "time") $(\mathrm{p}<0.001)$. In particular t-test between "groups" (i.e., patients vs. controls) showed that the blood lactate concentration before starting the exercise was significantly higher $(\mathrm{p}<0.001)$ in cancer patients compared to the controls (respectively $18.07 \pm$ 4.94 and $11.26 \pm 3.31 \mathrm{mg} / \mathrm{dl}$ ). This behaviour was maintained also at any given time during the recovery

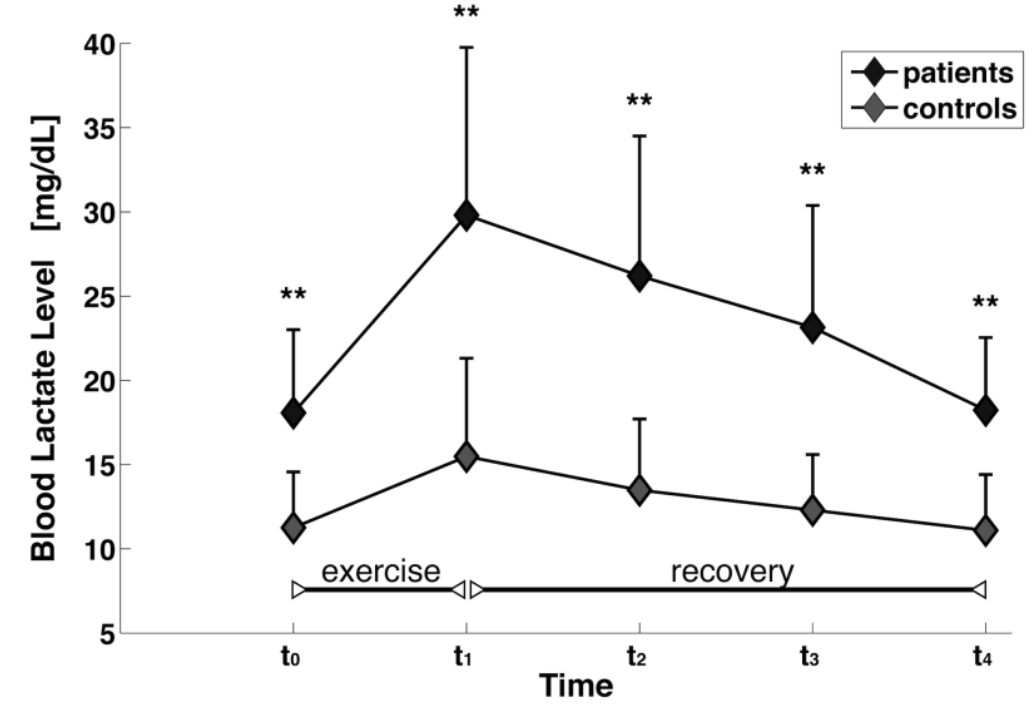

Fig 2. Blood concentration of lactate: Mean and one standard deviation of blood concentration of lactate, before $\left(t_{0}\right)$, at the end $\left(t_{1}\right)$, and after $\left(t_{2}=5^{\prime}, t_{3}=10^{\prime}, t_{4}=30^{\prime}\right.$ ) resistance exercise (an incremental sub-maximal aerobic exercise on a treadmill). Dark grey denotes oncologic patients, Light grey subjects of control group. The label ** highlights strong significant differences between healthy and patients $(p<0.001)$. 

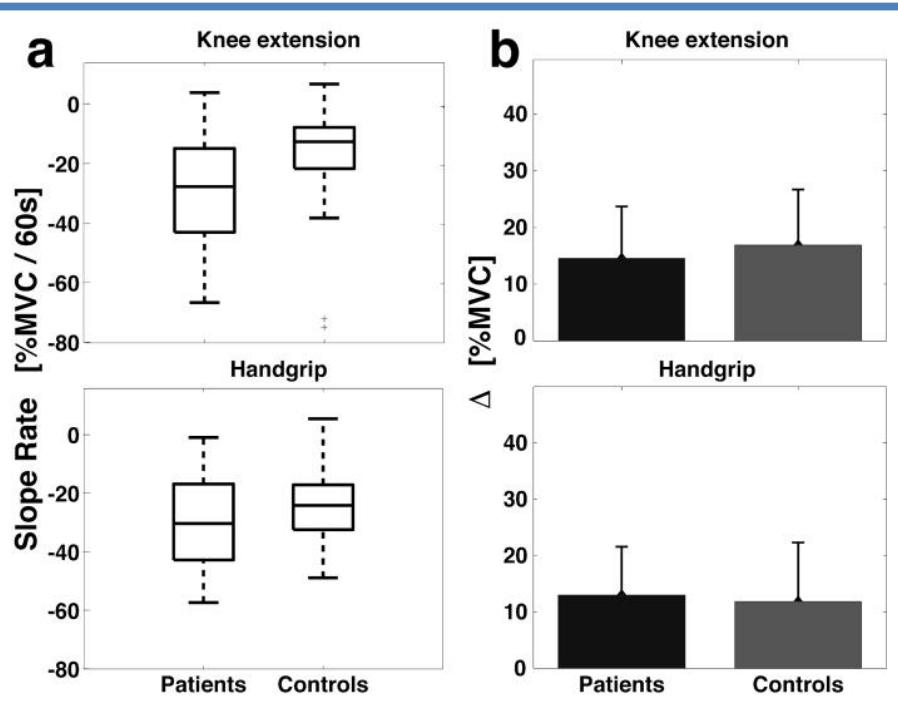

Fig 4. a: Box plot of muscle fatigue parameter $(\% M V C / 60 \mathrm{~s})$ for patients and healthy subjects. Top panel: knee extension. Bottom panel: handgrip. $b$ : Mean and one standard deviation of difference, in term of voluntary contraction normalized by Maximal Voluntary Contraction, between last $3 \mathrm{~s}$ of the endurance trial and the 3 $s$ after a short time (20 s) of rest, for patients (black) and healthy subjects (grey). Top panel: knee extension. Bottom panel: handgrip.

period (i.e., $\left.\mathrm{t}_{1}, \mathrm{t}_{2}, \mathrm{t}_{3}, \mathrm{t}_{4}\right)(\mathrm{p}<0.001)$ (Figure 2$)$. The mean values of the patients' maximal strength were $360.13 \pm$ $129.83 \mathrm{~N}$ for knee extension and $313.43 \pm 94.39 \mathrm{~N}$ for handgrip tasks. The mean values for healthy subjects were $423.59 \pm 160.35 \mathrm{~N}$ and $393.27 \pm 127.60 \mathrm{~N}$, (respectively for knee and handgrip tasks). Although there was a trend toward a lower maximum voluntary strength, in both lower and higher limbs, in patients compared to the control group, the statistical test did not reveal any significance (Figure 3). The fatigue test showed that the slope of the regression line (i.e., the percentage of decrement of holding strength) had greater values (without statistically significance) for patients than controls, both in knee and handgrip tasks (patients: $-27.84 \pm 19.45 \% \mathrm{MVC} / 60$ s, controls: $-16.29 \pm 19.02$ $\% \mathrm{MVC} / 60$ s for knee task; patients: $-29.93 \pm 15.62$ $\% \mathrm{MVC} / 60$ s, controls: $-25.59 \pm 11.64 \% \mathrm{MVC} / 60$ s for handgrip task) (Figure 4a). The $\Delta$ parameter highlight that patients and controls presented comparable values both for knee task (patients: $14.57 \pm 9.12$, controls: 16.90 \pm 10.23 ) and handgrip task (patients: $13.03 \pm 8.56$, controls: $11.89 \pm 10.44$ ) (Figure 4b).

\section{Discussion}

This study shows that cancer patients have a decreased muscle efficiency. They performed 15.7 minutes of treadmill walking while controls 19.6 minutes. This functional discrepancy is known from literature reporting a significant decline of spontaneous physical activity and weight in cancer patients, related to disease progression. ${ }^{33}$ However, in our patients, a significant weight loss was not observed. Moreover, considering the patient group, we observed a muscle strength reduction both in knee extension and handgrip performance and increased muscle fatigue compared to controls. It is known that skeletal muscle wasting and impaired muscle performance are prominent pathophysiological features of CC. Tumour-bearing animals and cancer patients display wasting, impaired muscle force production and acceleration onset of fatigue. ${ }^{34-35}$ Even if our results are not statistically significant, the relevance of the study is that our data were obtained on cancer patients who had no cachexia nor pre-cachexia and without any limitation in daily living activities. Furthermore, we highlighted an evidence of early-impaired muscle performance in patients, who did not show any weight loss or muscle wasting, but which have a very similar trend as regards the progressive decline of muscle strength and the earlier onset of fatigue as occurs in CC. The relevance of muscle fatigue in CC is well established: in fact fatigue is the most frequently reported symptom by cancer patients. Many of these patients perceive fatigue as the most distressing symptom associated with their illness because it imposes limitations on their physical activity level and in lifestyle. ${ }^{36}$ Skeletal muscle wasting, which occurs as part of $\mathrm{CC}$, is one of the mechanisms that contribute to fatigue. ${ }^{35}$ Patients enrolled in our study did not report fatigue as one of the prominent symptoms of the disease and did not report significant limitations in their daily living activities. However, more recently, Roberts and colleagues,${ }^{37}$ provided evidence that during severe CC muscle weakness in limb muscle cannot be entirely accounted for muscle atrophy. This indicates that muscle weakness is not just a consequence of muscle atrophy but that there is also significant contractile dysfunction. They also suggested that specifically targeting contractile dysfunction represents an additional means to counteract muscle weakness in $\mathrm{CC}$, in addition to targeting the 
prevention of muscle atrophy. According to these findings, we can assume that in our patients (nonexhibiting muscle wasting) a contractile dysfunction may be one of the mechanisms involved in impaired muscle performance. If true, we could think to design specific rehabilitative treatments, such as endurance exercise, to counteract cancer-related fatigue, as highlighted in many evidence. ${ }^{38-39}$ As regard muscle recovery, we observed a similar trend in the two groups. Analysis of this result must take into account that maximal contractions used in the present study depended primarily on anaerobic metabolism, so we can assume that in these patients this metabolic pathway is not affected. On the other hand, another interesting aspect investigated in this study was the efficiency of the aerobic metabolism of cancer patient's muscle. Analysis of blood lactate levels showed significantly higher values both at rest and in the recovery period in our subjects. This finding suggests a reduction of the oxidative muscle efficiency and a precocious resort to the anaerobic metabolism in these patients. It is well established that, during an aerobic prolonged exercise, lactate concentration lightly increases in blood. If the oxidative metabolism becomes insufficient, the energy output is guaranteed by anaerobic metabolism supplementation, and in this condition, lactate accumulates in the blood. ${ }^{40}$ This functional evidence, observed in our patients, is in accordance with literature. Actually, in a recent study, ${ }^{41}$ adenosine triphosphate (ATP) synthesis rate and the expression of genes that play key regulatory roles in skeletal muscle metabolism was examined in tumor-bearing mice by in vivo nuclear magnetic resonance: a reduced ATP synthesis rate and an atypical expression levels of these genes was shown, suggesting that reduced ATP synthesis is linked to mitochondrial dysfunction, ultimately leading to skeletal muscle wasting. ${ }^{41}$ Recently, a correlation between muscle wasting syndrome and mitochondrial dysfunction has been demonstrated in severely cachexia mice. In particular, the authors suggested that the expression of proteins regulating mitochondrial biogenesis and mitochondrial dynamics are disrupted early in the development of cachexia and precede a reduction in mitochondria content. Furthermore, alterations in the expression of these proteins are associated with higher levels of circulating IL-6. The protein expression was inhibited by the administration of an IL-6r antibody and was strongly down-regulated by exercise training. ${ }^{42}$ The early detection of oxidative defects in these patients may have extremely important therapeutic implications. In this regard, aerobic exercise could potentially be a promising intervention strategy for the prevention and treatment of CC. Different studies confirm the beneficial effect of aerobic training in diseases presenting impaired oxidative metabolism. Murphy et al. $^{43}$ showed an increase in muscle performance with reduced fatigue and lactate production in a group of patients with mitochondrial myopathies due to deletions of mitochondrial DNA after an aerobic training. Recently, our group demonstrated the effectiveness of an aerobic training in a patient with polymyositis in chronic phase with impairment of muscle oxidative metabolism. ${ }^{44}$ Mitochondria are dynamic organelles in skeletal muscle, critical in physical performance and disease. The mitochondrial life cycle spans biogenesis, maintenance, and clearance. Exercise training may promote each of these processes, conferring positive impacts on skeletal muscle contractile and metabolic functions. ${ }^{45}$ Finally, also the role of resistance exercise must be considered: resistance exercise has been demonstrated to be a potent stimulant for increasing protein synthesis and has been shown to reverse skeletal muscle wasting in other diseases, increasing muscle strength and lean body mass. ${ }^{46}$ Adaptations to endurance exercise include improved oxygen delivery to muscles and increased oxidative capacity. ${ }^{47}$ Although there is little evidence for the support of resistance training in $\mathrm{CC}$, the positive effects on lean muscle mass, strength and muscle function in other populations experiencing muscle wasting indicates that is an effective intervention for the attenuation of progressive muscle wasting. In cancer patients, there is evidence that physical exercise contributes to reduce fatigue, to improve $\mathrm{QoL}$ and to relieve many of the side effects experienced both during and after "neoplastic" managements. Hopefully, by implementing appropriate exercise interventions it might be possible to prevent the wasting typical of cachexia. However, our study does have some limitations. The first one is the small number of patients included in the analysis and the unbalanced ratio male/female, which makes further studies in a major number of patients mandatory. The second limitation is the inclusion of both metastatic and non-metastatic cancer patients. It is known that metastatic patients do have major problems in terms of lean muscle mass loss and, in general, are more prone to developing cachexia. On the other hand, even if the number of patients was limited and made up of both metastatic and non-metastatic patients, our patients did have similar clinical features and none of them referred any meaningful weight loss or other cachexia-related symptoms. All this considered, this might also be proof that muscle metabolism-related problems are a more widespread problem in cancer patients than what known from the literature and might be a precocious sign of further, more clinically relevant problems. Further studies should then be performed to confirm these hypotheses.

In conclusion, data emerging from this study highlight an impairment of muscle oxidative metabolism in cancer patients with no signs of pre-cachexia or cachexia. A trend of precocious fatigability and an impairment of muscle strength production were also observed. These data, if further independently confirmed, underline the relevance of an early assessment of muscle function in oncologic patients in order to early propose rehabilitative approaches that could counteract motor impairment and eventually to slow down and/or prevent cancer cachexia. 


\section{Muscle impairment in pre-cachexia cancer patients}

Eur J Transl Myol 30 (2): 258-267, 2020

\section{List of acronyms}

ACEi - Angiotensin-Converting Enzyme inhibitors

AGHR - acyl ghrelin

AHT - Arterial Hypertension

ANOVA - Analysis of Variance

ATP - adenosine triphosphate

AVP - arginine vasopressin

BAA - Beta adrenergic agonist

BMI - body mass index

CC - Cancer cachexia

COPD - Chronic Obstructive Pulmonary Disease

CT - chemotherapy

DGHR - des-acyl ghrelin

EDTA - Ethylene DiAmine Tetraacetic Acid

$\mathrm{F}$ - female

$\mathrm{H}$ - Hypercholesterolemia

IFN $\gamma$ - Interferon gamma

IL-6 - Interleukin 6

M - male

MAFbx - atrogin-1/muscle atrophy F-box

MuRF1 - Muscle RING Finger Protein 1

MVC - Maximum Voluntary Contraction

PIF - Proteolysis-Inducing Factors

QoL - Quality of Life

RT - Radiotherapy

ST - Surgical therapy

TNF $\alpha$ - Tumor Necrosis Factor alpha

\section{Authors contributions}

SD and CC designed the study. LG and AS enrolled patients. SD conducted the muscular function assessment and collected clinical data. PT performed data analysis. SD and CC contributed to data analysis and interpretation. SD drafted the manuscript. All authors read and approved the final version of the manuscript.

\section{Acknowledgments}

The authors wish to thank all the participants enrolled in the study for their time and contributions.

\section{Funding}

This work was supported from Italian Ministry of Education, Universities and Research (MIUR) (PRIN 2009/2011 to CC as coinvestigator).

\section{Conflict of Interest}

The authors declare they have no conflicts of interest.

\section{Ethical Publication Statement}

We confirm that we have read the Journal's position on issues involved in ethical publication and affirm that this report is consistent with those guidelines.

\section{Corresponding Author}

Dr. Stefania Dalise, Unit of Neurorehabilitation, University Hospital of Pisa, via Paradisa 2, 56124 Pisa, Italy. Phone; +39 050 996907; Fax: +39 050995723. ORCID iD: 0000-0002-1422-1539

E-mail: stefania.dalise@ao-pisa.toscana.it
E-mails of co-authors

Peppino Tropea: p.tropea@ccppdezza.it ORCID iD: 0000-0001-8238-9619

Luca Galli: lugal71@yahoo.it

ORCID iD: 0000-0001-6008-0266

Andrea Sbrana: andreasbrana89@gmail.com ORCID iD: 0000-0003-4460-5282

Carmelo Chisari: c.chisari@ao-pisa.toscana.it ORCID iD: 0000-0002-1043-7738

\section{References}

1. Tisdale MJ. Cachexia in cancer patients. Nat Rev Cancer 2002;2:862-71.

2. Dewys WD, Begg C, Lavin PT, et al. Prognostic effect of weight loss prior to chemotherapy in cancer patients. Eastern Cooperative Oncology Group. Am J Med 1980;69:491-7.

3. Evans WJ, Morley JE, Argiles J, et al. Cachexia: a new definition. Clin Nutr 2008;27:793-9.

4. Inagaki J, Rodriguez V, Bodey GP. Proceedings: Causes of death in cancer patients. Cancer 1974;33:568-73.

5. Le Bricon T, Gugins S, Cynober L, Baracos VE. Negative impact of cancer chemotherapy on protein metabolism in healthy and tumor-bearing rats. Metabolism 1995;44:1340-8.

6. Currow D, Temel JS, Abernethy A, et al. ROMANA 3: a phase 3 safety extension study of anamorelin in advanced non-small-cell lung cancer (NSCLC) patients with cachexia. Ann Oncol 2017;28:1949-56.

7. Temel JS, Abernethy AP, Currow DC, et al. Anamorelin in patients with non-small-cell lung cancer and cachexia (ROMANA 1 and ROMANA 2): results from two randomised, double-blind, phase 3 trials. Lancet Oncol 2016;17:519-31.

8. Argiles JM, Lopez-Soriano FJ. The role of cytokines in cancer cachexia. Med Res Rev 1999;19:223-48.

9. Reid MB, Li YP. Tumor necrosis factor-alpha and muscle wasting: a cellular perspective. Respir Res 2001;2:269-72.

10. Rennie MJ, Wackerhage H, Spangenburg EE, Booth FW. Control of the size of the human muscle mass. Annu Rev Physiol 2004;66:799-828.

11. Tisdale MJ. Molecular pathways leading to cancer cachexia. Physiology (Bethesda) 2005;20:340-8.

12. Zhao J, Brault JJ, Schild A, et al. FoxO3 coordinately activates protein degradation by the autophagic/lysosomal and proteasomal pathways in atrophying muscle cells. Cell Metab 2007;6:472-83.

13. Farges MC, Balcerzak D, Fisher BD, et al. Increased muscle proteolysis after local trauma mainly reflects macrophage-associated lysosomal proteolysis. Am J Physiol Endocrinol Metab 2002;282:E326-35. 


\section{Muscle impairment in pre-cachexia cancer patients}

Eur J Transl Myol 30 (2): 258-267, 2020

14. Hasselgren PO, Fischer JE. Muscle cachexia: current concepts of intracellular mechanisms and molecular regulation. Ann Surg 2001;233:9-17.

15. Jackman RW, Kandarian SC. The molecular basis of skeletal muscle atrophy. Am J Physiol Cell Physiol 2004;287:C834-43.

16. Lecker SH, Solomon V, Mitch WE, Goldberg AL. Muscle protein breakdown and the critical role of the ubiquitin-proteasome pathway in normal and disease states J Nutr 1999;129:227S-37S.

17. Temparis S, Asensi M, Taillandier D, et al. Increased ATP-ubiquitin-dependent proteolysis in skeletal muscles of tumor-bearing rats. Cancer Res 1994;54:5568-73.

18. Williams A, Sun X, Fischer JE, Hasselgren PO. The expression of genes in the ubiquitin-proteasome proteolytic pathway is increased in skeletal muscle from patients with cancer. Surgery 1999;126:744-9; discussion 9-50.

19. Bodine SC, Latres $\mathrm{E}$, Baumhueter $\mathrm{S}$, et al. Identification of ubiquitin ligases required for skeletal muscle atrophy. Science 2001;294:1704-8.

20. Gomes MD, Lecker SH, Jagoe RT, et al. Atrogin-1, a muscle-specific F-box protein highly expressed during muscle atrophy. Proc Natl Acad Sci U S A 2001;98:14440-5.

21. Glass DJ. Signalling pathways that mediate skeletal muscle hypertrophy and atrophy. Nat Cell Biol 2003;5:87-90.

22. Jackman RW, Kandarian SC. The molecular basis of skeletal muscle atrophy. American Journal of Physiology - Cell Physiology 2004;287:C834-C43.

23. Aulino P, Berardi E, Cardillo VM, et al. Molecular, cellular and physiological characterization of the cancer cachexia-inducing C26 colon carcinoma in mouse. BMC Cancer 2010;10:363.

24. Holmes MD, Chen WY, Feskanich D, et al. Physical activity and survival after breast cancer diagnosis. JAMA 2005;293:2479-86.

25. Meyerhardt JA, Giovannucci EL, Holmes MD, et al. Physical activity and survival after colorectal cancer diagnosis. J Clin Oncol 2006;24:3527-34.

26. Irwin ML, Smith AW, McTiernan A, et al. Influence of pre- and postdiagnosis physical activity on mortality in breast cancer survivors: the health, eating, activity, and lifestyle study. J Clin Oncol 2008;26:3958-64.

27. Fearon K, Strasser F, Anker SD, et al. Definition and classification of cancer cachexia: an international consensus. Lancet Oncol 2011;12:489-95.

28. Cupisti A, Licitra R, Chisari C, et al. Skeletal muscle and nutritional assessment in chronic renal failure patients on a protein-restricted diet. J Intern Med 2004;255:115-24.

29. Bertolucci F, Neri R, Dalise S, et al. Abnormal lactate levels in patients with polymyositis and dermatomyositis: the benefits of a specific rehabilitative program. Eur J Phys Rehabil Med 2013.

30. Pimentel AE, Gentile CL, Tanaka H, et al. Greater rate of decline in maximal aerobic capacity with age in endurance-trained than in sedentary men. J Appl Physiol (1985) 2003;94:2406-13.

31. Shechtman O. The coefficient of variation as a measure of sincerity of effort of grip strength, Part II: sensitivity and specificity. J Hand Ther 2001;14:188-94.

32. Kirk R. Experimental design: procedures for the behavioral sciences. Second ed. Monterey, Calif: Brooks/Cole Pub. Co; 1982.

33. Fouladiun M, Korner U, Gunnebo L, et al. Daily physical-rest activities in relation to nutritional state, metabolism, and quality of life in cancer patients with progressive cachexia. Clin Cancer Res 2007;13:6379-85.

34. Toledo M, Busquets S, Sirisi S, et al. Cancer cachexia: physical activity and muscle force in tumour-bearing rats. Oncol Rep 2011;25:189-93.

35. al-Majid S, McCarthy DO. Cancer-induced fatigue and skeletal muscle wasting: the role of exercise. Biol Res Nurs 2001;2:186-97.

36. Cella D, Davis K, Breitbart W, et al. Cancer-related fatigue: prevalence of proposed diagnostic criteria in a United States sample of cancer survivors. J Clin Oncol 2001;19:3385-91.

37. Roberts BM, Frye GS, Ahn B, et al. Cancer cachexia decreases specific force and accelerates fatigue in limb muscle. Biochem Biophys Res Commun 2013;435:488-92.

38. Khamoui AV, Kim JS. Candidate mechanisms underlying effects of contractile activity on muscle morphology and energetics in cancer cachexia. Eur J Cancer Care (Engl) 2012;21:143-57.

39. Puetz TW, Herring MP. Differential effects of exercise on cancer-related fatigue during and following treatment: a meta-analysis. Am J Prev Med 2012;43:e1-24.

40. Robergs RA, Ghiasvand F, Parker D. Biochemistry of exercise-induced metabolic acidosis. Am J Physiol Regul Integr Comp Physiol 2004;287:R502-16.

41. Constantinou C, Fontes de Oliveira CC, Mintzopoulos D, et al. Nuclear magnetic resonance in conjunction with functional genomics suggests mitochondrial dysfunction in a murine model of cancer cachexia. Int J Mol Med 2011;27:15-24.

42. White JP, Puppa MJ, Sato S, et al. IL-6 regulation on skeletal muscle mitochondrial remodeling during cancer cachexia in the ApcMin/+ mouse. Skelet Muscle 2012;2:14.

43. Murphy JL, Blakely EL, Schaefer AM, et al. Resistance training in patients with single, largescale deletions of mitochondrial DNA. Brain 2008;131:2832-40. 


\section{Muscle impairment in pre-cachexia cancer patients}

Eur J Transl Myol 30 (2): 258-267, 2020

44. Dalise S, Bertolucci F, Simonella C, et al. Intensive aerobic training improves motor performances and oxidative metabolism efficiency in chronic polymyositis: a case report. Neuromuscul Disord 2012;22 Suppl 3:S221-5.

45. Yan Z, Lira VA, Greene NP. Exercise traininginduced regulation of mitochondrial quality. Exerc Sport Sci Rev 2012;40:159-64.

46. Evans WJ, Roubenoff R, Shevitz A. Exercise and the treatment of wasting: aging and human immunodeficiency virus infection. Semin Oncol 1998;25:112-22.
47. Hakkinen A, Pakarinen A, Hannonen $\mathrm{P}$, et al. Effects of prolonged combined strength and endurance training on physical fitness, body composition and serum hormones in women with rheumatoid arthritis and in healthy controls. Clin Exp Rheumatol 2005;23:505-12.

Submitted: February, 28, 2019

Revision received: March 28, 2020 Accepted for publication: March 28, 2020 